\title{
Numerical investigation of vibrational relaxation coupling with turbulent mixing
}

\author{
Romain Fiévet*1 ${ }^{*}$ Stephen Voelkel ${ }^{\dagger 2}$, Venkat Raman ${ }^{\ddagger 1}$ and Philip Varghese ${ }^{\S 2}$ \\ ${ }^{1}$ Department of Aerospace Engineering, University of Michigan, Ann Arbor, MI 48109-2102, USA \\ ${ }^{2}$ Institute for Computational Engineering and Sciences, The University of Texas at Austin, Austin, TX-78712, USA
}

\begin{abstract}
In flows where the relaxation rate of vibrational motion of the molecules to equilibrium is comparable to the flow through time scales, the presence of turbulence can alter the mixing and equilibration process. To understand the coupling between mixing and vibrational relaxation, a novel state-specific species model is solved in a background turbulent flow. The method is applied to mixing of two nitrogen streams at different static temperatures. The relaxation rates for each state are computed using quasi-classical trajectory analysis. For the flow conditions considered, the first ten vibrational levels are computed in the flow solver. The direct numerical simulation shows that population in different vibrational levels are significantly affected by turbulence and that the local distribution becomes nonBoltzmann. In certain locations in the jet, the population from the direct calculation can be several orders of magnitude different than the local-temperature based Boltzmann level. Last, while the bulk vibrational energy is inferior to its local equilibrium value throughout the mixing layer, the high energy level populations (levels 3 to 8 ) are on the opposite always over-populated. As chemical reactions are affected by these high vibrational energy populations, a simple temperature model would under-estimate the impact of nonequilibrium on combustion.
\end{abstract}

\section{Introduction}

The impact of vibrational nonequilibrium on mixing and chemical reaction rates has been extensively studied. ${ }^{1-6}$ These models have been used to study configurations exhibiting strong levels of nonequilibrium, typically high-speed shock-containing flows, such as supersonic combustion ramjet (scramjet). ${ }^{6-9}$ In scramjet engines, the flow is thrown out of equilibrium as it passes through a series of compression shocks in the isolator, expansion waves at the fuel injectors, and turbulent mixing. The short residence time means that ignition will happen while the vibrational relaxation process is still ongoing. This proves to be a design challenge as fast ignition and combustion efficiency provides the vehicle thrust, which determines in turns the compression ratio at the combustor entrance. The failure of one component results in engine unstart. Similarly, in a rotating detonating engine (RDE), ignition must happen fast enough to sustain the detonation wave propagation speed and compression ratio. It happens right behind the detonation front, where the higher vibrational energy states are under-populated. Interestingly, vibrational nonequilibrium is not only caused by the strong temperature jump at shock wave fronts, but is also present upstream due to turbulent mixing between the cold fuel expanded from the pressurized tank and the hot air. Fiévet et al. ${ }^{6}$ showed that vibrational nonequilibrium can drastically affect ignition time in a scramjet engine while Taylor et al. ${ }^{10}$ postulated that it influences the detonation cells structure. Its impact on the flow is also consequent

${ }^{*}$ PhD Student, University of Michigan, AIAA Student Member.

${ }^{\dagger}$ PhD Student, The University of Texas at Austin, AIAA Student Member.

$¥$ Associate Professor, University of Michigan, AIAA Associate Fellow,

$\S$ Professor, The University of Texas at Austin, AIAA Associate Fellow,

Copyright (C) 2016 by Romain Fiévet. Published by the American Institute of Aeronautics and Astronautics, Inc. with permission. 
prior to ignition, as internal energy exchanges with the vibrational modes will change the flow translational temperature, affecting the bulk static pressure, temperature, and diffusive processes. Accurate numerical description of this phenomenon is therefore a key element of a compressible solver used in such environment and serves as motivation for the current study.

While post-compression/expansion wave nonequilibrium and relaxation process can be studied independently of turbulence phenomena in a 1D configuration, the free shear flow offers more complex yet canonical configuration. In this case there is a close coupling between turbulence mixing and these quantum effects, affecting macroscopic flow properties (shear layer shape, potential core length, mixture fraction), and therefore eventual chemical reactions further downstream. The current study aims at investigating such a flow by the mean of a Direct Numerical Simulation (DNS), where all turbulent scales are resolved, coupled with a vibrational states distribution solver. The solver carries a set of scalars corresponding to the vibrational states number densities relevant to the temperature range throughout the computational domain. The simulations will focus on a non-reacting single-specie free shear flow where translational and rotational modes are assumed to be in thermodynamic equilibrium, i.e. their states distributions follows a Boltzmann distribution computed from the same temperature. This is a reasonable assumption as these modes reach equilibrium within orders of magnitude less collisions than the vibrational mode. Most studies previously cited ${ }^{1-4,6-9}$ use a multi-temperature approach to describe vibrational nonequilibrium: the vibrational states distribution is considered to remain Boltzmann at a different temperature than the translational temperature. This relies on the assumption that the relaxation timescales are identical for all energy states. However, as the vibrational quantum number increases, the state-to-state energy difference decreases, resulting in a much faster relaxation process at higher energy levels, effectively distorting the distribution from its original Boltzmann shape. This error grows as higher vibrational energy levels are populated for hotter flows, and can lead to erroneous reaction rates estimation and translational temperature as well. This last point is crucial in determining the local gas kinetic viscosity, and therefore accurately resolving turbulence structures. Two models will be used to evaluate the distribution relaxation process. First, a modified Landau \& Teller ${ }^{11}$ linear relaxation model with a common relaxation timescale for all states is considered, similar to the multi-temperature approach previously described. Second, a quasi-classical trajectory (QCT) model is used to calculate statespecific population rates, in an effort to capture the complexity of the vibrational relaxation process through molecular inelastic collisions, allowing the description of non-Boltzmann distribution throughout the flow.

The paper is organized as follow : a first section will present the different models implemented to numerically describe vibrational relaxation, with a strong focus on the novel state-specific rates model introduced in the current work. The second section will present the compressible flow solver and numerical details of the configuration. A third part presents the results and observations.

\section{Derivation of state-specific vibrational relaxation rates}

\section{A. QCT formulation of inelastic rates}

The vibrational inelastic rates for the reaction

$$
\mathrm{N}_{2}\left(v_{1}\right)+\mathrm{N}_{2}\left(v_{2}\right) \longrightarrow \mathrm{N}_{2}\left(v_{1}^{\prime}\right)+\mathrm{N}_{2}\left(v_{2}^{\prime}\right)
$$

were calculating using quasi-classical trajectory (QCT) analysis. ${ }^{5,12,13}$ The QCT method assumes that the motion of the nuclei are classical and driven by the potential energy surface (PES). For a particular trajectory, the $i^{\text {th }}$ nuclei follows a classical path governed by

$$
\begin{aligned}
\frac{d \boldsymbol{Q}_{i}}{d t} & =\boldsymbol{P}_{i} \\
\frac{d \boldsymbol{P}_{i}}{d t} & =-\frac{\partial V}{\partial \boldsymbol{Q}_{i}},
\end{aligned}
$$

where $\boldsymbol{Q}_{i}$ and $\boldsymbol{P}_{i}$ are the position and momentum vectors of the $i^{\text {th }}$, respectively, and $V$ represents the PES. Solving these classical equations is the main process in a QCT simulation. The numerical method chosen to solve Eqs. 2 and 3 was the adaptive Runge-Kutta Prince-Dormand $(8,9)$ method as implemented in the open source GNU Scientific Library (GSL). ${ }^{14}$ Though the method is not symplectic, the error tolerance was set sufficiently low so that the total energy of the system did not drift noticeably over a typical trajectory time frame. 
The inelastic scattering rates were approximately determined using Monte-Carlo integration as conventionally done in QCT analysis. $5,12,13$ Here, the rates were determined a function of a translationalrotational temperature $T$, an initial vibrational quantum number $\boldsymbol{v}=\left(v_{1}, v_{2}\right)$, and a final vibrational number $\boldsymbol{v}^{\prime}=\left(v_{1}^{\prime}, v_{2}^{\prime}\right)$. The scattering rate is denoted as $k_{s}\left(\boldsymbol{v}, \boldsymbol{v}^{\prime}, T\right)$. So, for each trajectory, $\boldsymbol{v}$ is fixed, and the relative speed and initial rotation quantum numbers are sampled based on their respective probability distribution functions (PDFs). After the $\mathrm{N}_{2}$ molecules collide, the final state is marked (i.e., $\boldsymbol{v}^{\prime}$ ). The aggregation of the outcomes is used to determine the inelastic scattering rates. The following section describes the process for calculated $k_{s}$ used for this $\mathrm{N}_{2}-\mathrm{N}_{2}$ system.

\section{Inelastic scattering rate calculation}

Each trajectory is initialized as follows. For the $i^{\text {th }}$ reactant, the motion is characterized by: (1) two angles to represent the initial orientation of the diatom, (2) a vibration phase based on $\boldsymbol{v}$, and (3) a rotation vector based on the initial rotational quantum number $\boldsymbol{J}=\left(J_{1}, J_{2}\right)$. Let $\boldsymbol{\tau}$ denote the initial orientation, vibration phase, and rotational vector of the reactants. The relative motion is characterized by: (1) the relative speed of the reactants $g,(2)$ the impact parameter $b$, and (3) the initial separation between the centers of mass of each reactant. The separation was set to be sufficiently large so that at the start of each trajectory, each reactant imposed negligible effects on the other (greater than $15 \AA$ for this system ${ }^{15}$ ).

For a given $\tau, g$, and $b$, the outcome of the trajectory is deterministic (i.e., only one outcome is observed). It follows that the outcome is a Dirac-delta function given by $\delta_{s}\left(\boldsymbol{v} \rightarrow \boldsymbol{v}^{\prime}, \boldsymbol{J} \rightarrow \boldsymbol{J}^{\prime}, g, b, \boldsymbol{\tau}\right)$, where $\delta_{s}=1$ if $\boldsymbol{v}^{\prime}$ and $\boldsymbol{J}^{\prime}$ are the final rovibrational quantum numbers and 0 otherwise (note that $\boldsymbol{v}$ and $\boldsymbol{J}$ are parameters from which $\tau$ is randomly sampled). The scattering probability $P_{s}$ is defined by averaged $\delta_{s}$ along $\boldsymbol{\tau}$, i.e.,

$$
P_{s}\left(\boldsymbol{v} \rightarrow \boldsymbol{v}^{\prime}, \boldsymbol{J} \rightarrow \boldsymbol{J}^{\prime}, g, b\right)=\int_{\boldsymbol{\tau}} \delta_{s}\left(\boldsymbol{v} \rightarrow \boldsymbol{v}^{\prime}, \boldsymbol{J} \rightarrow \boldsymbol{J}^{\prime}, g, b, \boldsymbol{\tau}\right) f_{\boldsymbol{\tau}}(\boldsymbol{\tau}) d \boldsymbol{\tau}
$$

where $f_{\boldsymbol{\tau}}(\boldsymbol{\tau}) d \boldsymbol{\tau}$ is the PDF of the initial orientations, vibration phases, and rotational vectors. This integral is approximating by Monte-Carlo integration. ${ }^{16}$ That is, for $N$ trajectories sampled at fixed $(\boldsymbol{v}, \boldsymbol{J}, g, b)$ and random $\boldsymbol{\tau}$ (sampled from its PDF), let $N_{s}$ denote the number of trajectories with a post-collision rovibrational quantum numbers $\boldsymbol{v}^{\prime}$ and $\boldsymbol{J}^{\prime}$. Then, $P_{s}$ is approximated by

$$
P_{s}\left(\boldsymbol{v} \rightarrow \boldsymbol{v}^{\prime}, \boldsymbol{J} \rightarrow \boldsymbol{J}^{\prime}, g, b\right) \approx \frac{N_{s}\left(\boldsymbol{v}^{\prime}, \boldsymbol{J}^{\prime}\right)}{N(\boldsymbol{v}, \boldsymbol{J}, g, b)},
$$

The relative uncertainty of $P_{s}$, denoted by $\varepsilon_{s}$, is defined as two standard deviations normalized by $P_{s}$ (this corresponds to a $95 \%$ confidence interval), which is given by ${ }^{5,12,13}$

$$
\varepsilon_{s}=2\left(\frac{1}{N_{s}}-\frac{1}{N}\right)^{1 / 2} \approx 2\left(\frac{1}{N_{s}}\right)^{1 / 2}
$$

where dependencies were dropped for brevity and the approximation is valid when $N \gg N_{s}$. So, for a $95 \%$ confidence interval to be within $5 \%$ of the mean (i.e., $\varepsilon=0.05$ ), approximately 1,600 trajectories in which $\boldsymbol{v} \rightarrow \boldsymbol{v}^{\prime}$ need to be observed.

The inelastic scattering cross-section $\sigma_{s}$ is calculated by integrating $P_{s}$ along $b$ weighted by $2 \pi b$, i.e.

$$
\sigma_{s}\left(\boldsymbol{v} \rightarrow \boldsymbol{v}^{\prime}, \boldsymbol{J} \rightarrow \boldsymbol{J}^{\prime}, g\right)=\int_{0}^{b_{\max }} 2 \pi b P_{s}\left(\boldsymbol{v} \rightarrow \boldsymbol{v}^{\prime}, \boldsymbol{J} \rightarrow \boldsymbol{J}^{\prime}, g, b\right) d b .
$$

where $P_{s}=0$ for all $b>b_{\max }$. As with the probability, the integral defining the cross-section is approximated by Monte-Carlo integration. ${ }^{16}$ By this, Eq. 7 is approximated as

$$
\sigma\left(\boldsymbol{v} \rightarrow \boldsymbol{v}^{\prime}, \boldsymbol{J} \rightarrow \boldsymbol{J}^{\prime}, g\right) \approx \pi b_{\max }^{2} \frac{N_{s}\left(\boldsymbol{v}^{\prime}, \boldsymbol{J}^{\prime}\right)}{N(\boldsymbol{v}, \boldsymbol{J}, g)}
$$

where now $N$ refers to trajectories wherein $\tau$ and $b$ are each randomly sampled from their respective PDFs. Note that the relative uncertainty of $\sigma_{s}$ is the same as defined in Eq. 6. 
The inelastic scattering rate $k_{s}$ is calculated by integrating $\sigma_{s}$ along $g$ weighted by $g f_{g}$, where $f_{g}$ is the PDF of the relative speed. Here, the translational energy is assumed to be in equilibrium, so the relative speed is characterized by a Maxwell distribution at temperature $T$ given by

$$
f_{g}(g ; T)=\left(\frac{\mu}{2 \pi k_{B} T}\right)^{3 / 2} 4 \pi g^{2} e^{-\mu g^{2} / 2 k_{B} T},
$$

where $\mu$ is the reduced mass of the reactants and $k_{B}$ is the Boltzmann constant. Then, $k_{s}$ is given by

$$
k_{s}\left(\boldsymbol{v} \rightarrow \boldsymbol{v}^{\prime}, \boldsymbol{J} \rightarrow \boldsymbol{J}^{\prime}, T\right)=\int_{0}^{\infty} g f_{g}(g ; T) \sigma\left(\boldsymbol{v} \rightarrow \boldsymbol{v}^{\prime}, \boldsymbol{J} \rightarrow \boldsymbol{J}^{\prime}, g\right) d g,
$$

As with the probability and cross-section, the integral defining the rate is approximated by Monte-Carlo integration. ${ }^{16}$ By this, Eq. 10 is approximated as

$$
k_{s}\left(\boldsymbol{v} \rightarrow \boldsymbol{v}^{\prime}, \boldsymbol{J} \rightarrow \boldsymbol{J}^{\prime}, T\right) \approx \pi b_{\max }^{2}\left(\frac{8 k_{B} T}{\pi \mu}\right)^{1 / 2} \frac{N_{s}\left(\boldsymbol{v}^{\prime}, \boldsymbol{J}^{\prime}\right)}{N(\boldsymbol{v}, \boldsymbol{J})},
$$

where now $N$ refers to trajectories wherein $\tau, b$, and $g$ are each randomly sampled from their respective PDFs. This denotes the rovibrationally state-specific inelastic scattering rate.

Now, the rate is averaged along the rotational states. As with the translational energy, the rotational energy is assumed to be in equilibrium, so the rotational energy is characterized by a Boltzmann distribution at temperature $T$. For the $i^{t h}$ reactant at a fixed vibrational quantum number $v_{i}$, this rotational energy $\mathrm{PDF}$ is defined as

$$
f_{r, i}\left(J_{i} ; v_{i}, T\right)=\frac{g_{s}\left(J_{i}\right)\left(2 J_{i}+1\right) e^{-\left(\epsilon_{\text {int }}\left(v_{i}, J_{i}\right)-\epsilon_{\text {int }}\left(v_{i}, 0\right)\right) / k_{B} T}}{Q_{r, i}\left(v_{i}, T\right)},
$$

where $g_{s}$ is the spin degeneracy of the rotational state, $\epsilon_{\text {int }}$ is the internal energy, and $Q_{r}$ is the rotational partition function, which normalizes $f_{r, i}$. The combined rotational PDF is denoted $f_{r}(\boldsymbol{J} ; \boldsymbol{v}, T)=$ $f_{r, 1}\left(J_{1} ; v_{1}, T\right) f_{r, 2}\left(J_{2} ; v_{2}, T\right)$. Using this PDF, the vibrationally state-specific rate is given by

$$
k_{s}\left(\boldsymbol{v} \rightarrow \boldsymbol{v}^{\prime}, T\right)=\sum_{\boldsymbol{J}} \sum_{\boldsymbol{J}^{\prime}} f_{r}(\boldsymbol{J} ; \boldsymbol{v}, T) k_{s}\left(\boldsymbol{v} \rightarrow \boldsymbol{v}^{\prime}, \boldsymbol{J} \rightarrow \boldsymbol{J}^{\prime}, T\right)
$$

Similar to before, the Monte-Carlo method approximates Eq. 7 as $^{16}$

$$
k_{s}\left(\boldsymbol{v} \rightarrow \boldsymbol{v}^{\prime}, T\right) \approx \pi b_{\max }^{2}\left(\frac{8 k_{B} T}{\pi \mu}\right)^{1 / 2} \frac{N_{s}\left(\boldsymbol{v}^{\prime}\right)}{N(\boldsymbol{v})}
$$

where now $N$ refers to trajectories wherein $\boldsymbol{\tau}, b, g$, and $\boldsymbol{J}$ are each randomly sampled from their respective PDFs, and $N_{s}$ does not count the final rotational quantum numbers. Note that the relative uncertainty of $k_{s}$ is the same as defined in Eq. 6 . Finally, note that $k_{s}$ is divided by two to account for the fact that both reactants are the same species.

The rates as defined in Eq. 14 are completely independent from one another. Thus, the QCT-calculated rates are not necessarily symmetric and detailed balance at thermal equilibrium is not guaranteed. To ensure that the rates are consistent (i.e., the rates are symmetric and give rise to detailed balance), the rates are modified as necessary. First, for symmetry, the rates are modified so that

$$
k_{s}\left(\left(v_{1}, v_{2}\right) \rightarrow\left(v_{1}^{\prime}, v_{2}^{\prime}\right), T\right)=k_{s}\left(\left(v_{2}, v_{1}\right) \rightarrow\left(v_{2}^{\prime}, v_{1}^{\prime}\right), T\right)
$$

This relation is imposed directly in Eq. 14 by setting

$$
\begin{aligned}
N_{s}(\boldsymbol{v}) & =N\left(v_{1}, v_{2}\right)+N\left(v_{2}, v_{1}\right) \\
N_{s}\left(\boldsymbol{v} \rightarrow \boldsymbol{v}^{\prime}\right) & =N_{s}\left(v_{1} \rightarrow v_{1}^{\prime}, v_{2} \rightarrow v_{2}^{\prime}\right)+N_{s}\left(v_{2} \rightarrow v_{2}^{\prime}, v_{1} \rightarrow v_{1}^{\prime}\right) .
\end{aligned}
$$

Note that $\boldsymbol{v}$ is added as a dependency for $N_{s}$ to be clarify how final states are counted. Next, for detailed balance, the rates are modified so that

$$
f_{v}(\boldsymbol{v}, T) k_{s}\left(\boldsymbol{v} \rightarrow \boldsymbol{v}^{\prime}, T\right)=f_{v}\left(\boldsymbol{v}^{\prime}, T\right) k_{s}\left(\boldsymbol{v}^{\prime} \rightarrow \boldsymbol{v}, T\right)
$$


where $f_{v}$ is a Boltzmann function that characterizes the vibrational energy distribution. This relation is imposed by calculated a set of detailed balance rates from the QCT-calculated rates, i.e.,

$$
k_{s}^{*}\left(\boldsymbol{v} \rightarrow \boldsymbol{v}^{\prime}, T\right)=\frac{f_{v}\left(\boldsymbol{v}^{\prime}, T\right)}{f_{v}(\boldsymbol{v}, T)} k_{s}\left(\boldsymbol{v}^{\prime} \rightarrow \boldsymbol{v}, T\right) .
$$

Then, a final set of rates is defined as $\frac{1}{2}\left(k_{s}+k_{s}^{*}\right)$ for use in the CFD simulation.

\section{B. State-specific relaxation rates}

Inelastic scattering rates were directly calculated using the QCT method as derived in Sec. II at 9 translationalrotational temperatures:

$$
T=500,1,000,1,500,2,000,2,500,3,000,4,000,5,000 \text {, and 6,000 K. }
$$

The first 10 vibrational quantum numbers were sampled, so for $\mathrm{N}_{2}-\mathrm{N}_{2}$ collisions, there exist $10^{4}$ vibrational state combinations (i.e, degrees of freedom). A total of 2.8 billion trajectories were simulated using the QCT program developed by Voelkel et al. ${ }^{5,13}$ on the Texas Advanced Computing Center (TACC) machine using 4,104 cores for 30 hours. At the end of each trajectory, the final vibrational quantum number was determined as the closest lying state compared to the classical vibrational energy resulting from the collision.

In total, $9 \times 10^{4}$ rates were to be calculated based on the sampled states (including both inelastic and elastic collisions). However, many of the final states were never observed, implying that the probability of the particular transition was approximately zero. Table 1 summarizes the number of trajectories and rates calculated per temperature. Fewer transitions were observed at lower temperatures (resulting in fewer

Table 1: Number of trajectories and rates calculated per sampled temperature.

\begin{tabular}{ccc}
\hline \hline Temperature $(\mathrm{K})$ & Trajectories & Calculated Rates \\
\hline 500 & $2.3 \times 10^{8}$ & 438 \\
1,000 & $2.3 \times 10^{8}$ & 468 \\
1,500 & $2.3 \times 10^{8}$ & 480 \\
2,000 & $2.3 \times 10^{8}$ & 526 \\
2,500 & $1.6 \times 10^{7}$ & 512 \\
3,000 & $2.3 \times 10^{8}$ & 877 \\
4,000 & $6.3 \times 10^{8}$ & 2,493 \\
5,000 & $5.0 \times 10^{8}$ & 4,344 \\
6,000 & $5.0 \times 10^{8}$ & 6,124 \\
\hline \hline
\end{tabular}

calculated rates) because the total energy of the colliding $\mathrm{N}_{2}-\mathrm{N}_{2}$ pair was not sufficient to dramatically shift the vibrational state. At high temperatures and vibrational quantum numbers, more energy is stored in translational-rotational and vibrational energy modes on average. This increases the total energy that can be repartitioned during the collision event, which in turn increases the likelihood of observing vibrational transitions.

To illustrate the difficulty of observing transitions at low-lying vibrational states and at low temperatures, consider the transition from $\left(v_{1}=0, v_{2}=0\right)$ to $\left(v_{1}^{\prime}=1, v_{2}^{\prime}=0\right)$. For $\mathrm{N}_{2}$, at $500 \mathrm{~K}$ the most probable rovibrational energy to be sampled is $0.165 \mathrm{eV}$, and the most probable translational energy to be sampled is $0.043 \mathrm{eV}$, corresponding to an initial total energy of $0.373 \mathrm{eV}$. Assuming that all translational-rotational energy can be converted into vibrational energy, there exists $0.079 \mathrm{eV}$ of available energy. However, the energy difference between $v=0$ and $v=1$ is approximately $0.3 \mathrm{eV}$, so at this temperature, the translationalrotational energy is not sufficient to cause a transition. In contrast, using a similar analysis at 4,000 K, there exists $0.690 \mathrm{eV}$ of translational-rotational energy, which is sufficient to increase the vibrational energy from $v=0$ to $v=1$. Thus, it is far more likely that this transition will be observed.

The set of directly calculated inelastic rates, at $2,000 \mathrm{~K}$ and $4,000 \mathrm{~K}$ is plotted in Fig. 1, and the corresponding plots are referred to as the rate matrix for a particular temperature. The $x$ and $y$ axes correspond to the initial/final state of the first and second nitrogen molecule, respectively. Because symmetry was enforced, the rate matrix is symmetric across the $x=y$ diagonal. Note that if the rate is zero, this implies 


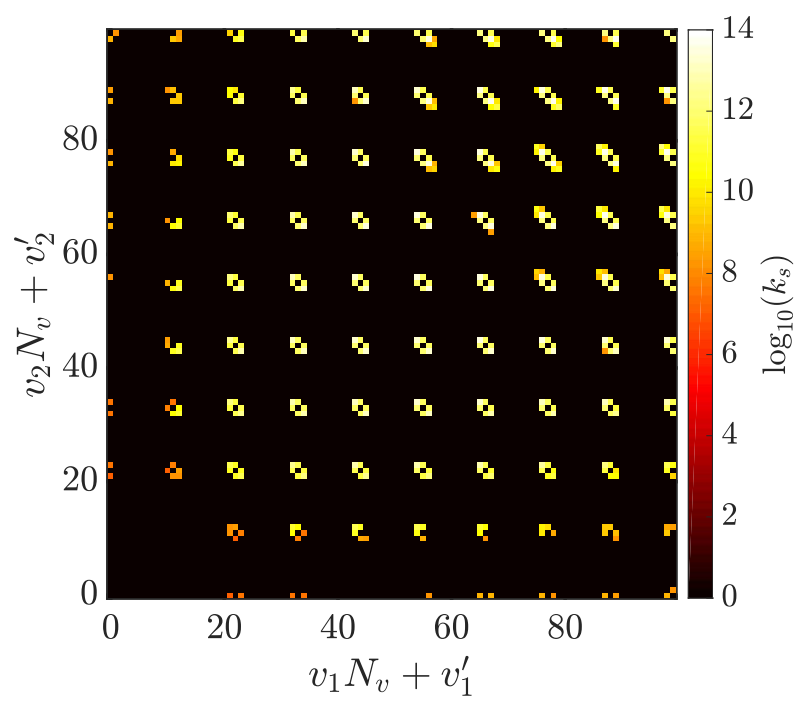

(a) $T=2,000 \mathrm{~K}$

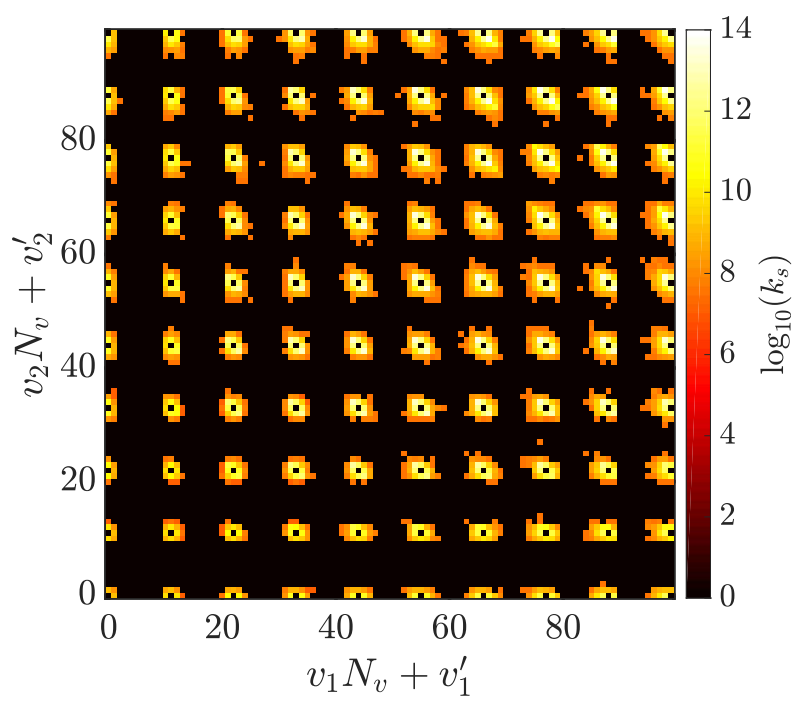

(b) $T=4,000 \mathrm{~K}$

Figure 1: Directly calculated inelastic scattering rates (units for the rate are $\mathrm{cm}^{3} / \mathrm{mol} / \mathrm{s}$ ).

that the transition was not recorded throughout the QCT simulation. Hence, the rate matrix at 2,000 K is sparse because most vibrational state transitions were not observed, whereas the rate matrix at 4,000 K is less sparse. Note that the rates at the other temperatures follow the general trends seen for these two temperatures.

In Fig. 1a, the directly calculated rates for low-lying vibrational states is zero, but the physical rate is non-zero. This discrepancy is due to the statistical nature of the QCT method and the low probability of observing such a transition. To overcome this, the scattering rates at low temperatures were extrapolated from the rates at high temperatures. Furthermore, from the set of directly calculated rates, as modified to the enforce detailed balance (Eq. 19), the scattering rates were also interpolated at intermediate temperatures between $1,800 \mathrm{~K}$ and $4,200 \mathrm{~K}$ at an increment of $2 \mathrm{~K}$. For the interpolation, it was assumed that the logarithm of $k_{s}$ was proportional to the inverse of $T$ (similar to the conventional Arrhenius expression). The interpolated (and extrapolated) rate matrices at 2,000 K and 4,000 K are plotted in Fig. 2. Comparing Fig. 2b with Fig.1b,

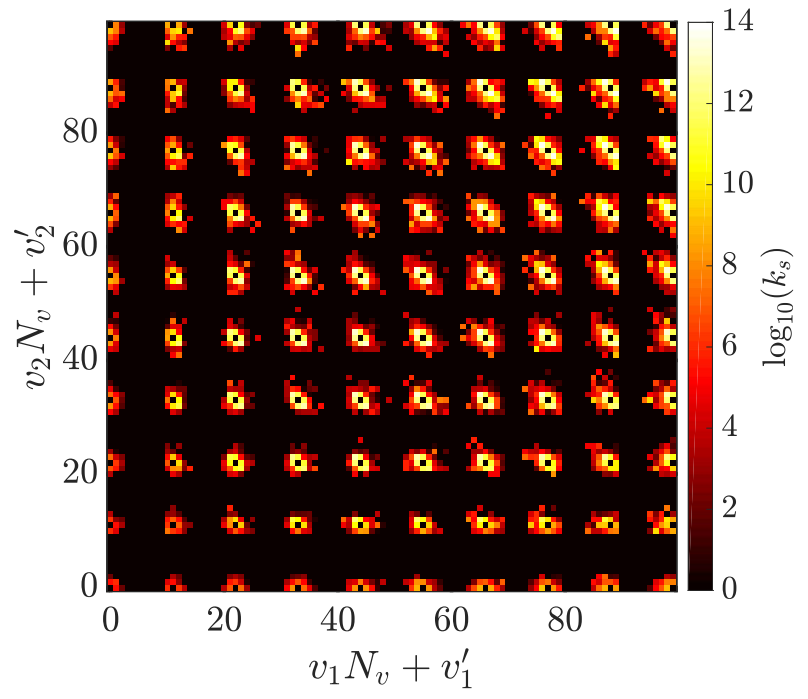

(a) $T=2,000 \mathrm{~K}$

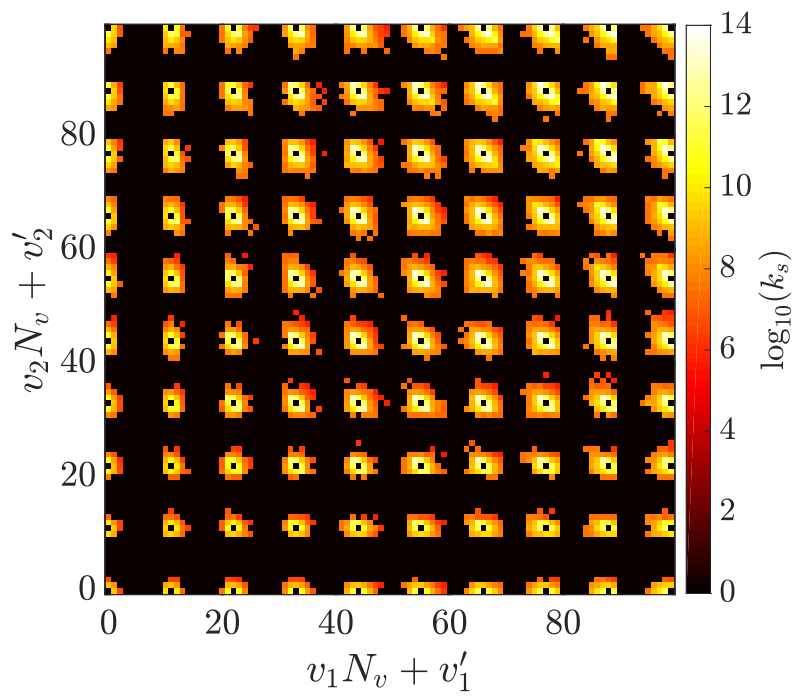

(b) $T=4,000 \mathrm{~K}$

Figure 2: Interpolated and extrapolated inelastic scattering rates (units for the rate $\operatorname{are~} \mathrm{cm}^{3} / \mathrm{mol} / \mathrm{s}$ ).

notice that the rate matrix for $4,000 \mathrm{~K}$ is approximately unchanged. However, at 2,000 K, the interpolated rate matrix is significantly less sparse than before. Specifically, the non-zero entries in the rate matrix was increased from 526 to 2,521. These interpolated rate matrices were used in the thermal bath and turbulent 
planar jet simulations.

\section{Thermal bath simulations}

As an initial test, the rates were used to simulate vibrational relaxation on $\mathrm{N}_{2}$ in a $0 \mathrm{D}$ heat bath. Here, for a fixed translational-rotational temperature $T$, the evolution of the number density of the $i^{\text {th }}$ vibrational state $\phi_{i}$ is governed by

$$
\frac{d \phi_{i}}{d t}=\sum_{j} \sum_{k} \sum_{l} g_{i j k l}\left(\phi_{k} \phi_{l} k_{s}\left(v_{k} \rightarrow v_{i}, v_{l} \rightarrow v_{j}, T\right)-\phi_{i} \phi_{j} k_{s}\left(v_{i} \rightarrow v_{k}, v_{j} \rightarrow v_{l}, T\right)\right)
$$

where the summations over $j, k$, and $l$ are for all vibrational states, and $g_{i j k l}$ is a degeneracy factor given by

$$
g_{i j k l}=\left(1+\delta_{i j}\left(1-\delta_{i k}\right)\left(1-\delta_{i l}\right)\right)\left(1-\delta_{i k}\left(1-\delta_{i j}\right)\right)\left(1-\delta_{i l}\left(1-\delta_{i j}\right)\right) .
$$

The initial vibrational populations were set based on a Boltzmann distribution is some $T_{v}$. Then, Eq. 21 was solved until the final vibrational state matched a Boltzmann distribution at the translational-rotational temperature $T$.

Two cases were tested: (1) $T_{v}<T$ (cold to hot), and (2) $T_{v}>T$ (hot to cold). In both cases, $T$ was fixed throughout the simulation, and the pressure was fixed at $1 \mathrm{~atm}$. Figure 3 shows the normalized energy versus time for cold-to-hot (red) and hot-to-cold (blue) relaxation processes In general, the cold-to-hot relaxation

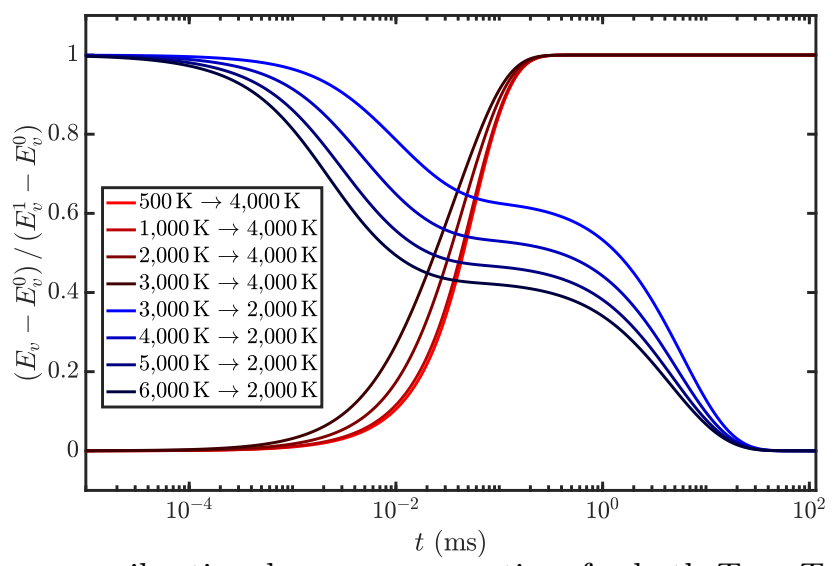

Figure 3: Average vibrational energy versus time for both $T_{v}<T$ and $T_{v}>T$

process for all temperature combinations is well characterized by a Landau-Teller model ${ }^{11}$ (i.e., exponential relaxation). From hot-to-cold, this is not the case. Instead, a quasi-steady state is achieved quickly during the relaxation process, which then relaxes more slowly towards equilibrium. It was observed that the initial quasi-steady state corresponded to the relaxation of high-lying vibrational states, and the final steady state was governed largely by the low-lying vibrational states.

In Fig. 4, the change in vibrational relaxation based on the initial vibrational distribution was measured for several temperatures. The figure shows that as the temperature increases, the relaxation time decreases. This is attributed to two main causes: (1) as the temperature increases, the inelastic scattering rates also increase (see Fig. 2), and (2) as the temperature increases, the collision rate increases. The figure also suggests that the initial distribution only plays a role in the relaxation process at lower temperatures. At $6,000 \mathrm{~K}$, the difference in the relaxation processes is marginal, whereas at $3,000 \mathrm{~K}$, the relaxation from $500 \mathrm{~K}$ takes approximately an order of magnitude longer to begin compared to the relaxation from 2,000 K. However, the time to reach a final steady state is approximately independent of the initial distribution, regardless of the final temperature. This suggests the relaxation model can be parametrized by only the translational-rotational temperature, as done by Millikan and White. ${ }^{17}$

\section{Compressible flow solver with vibrational nonequilibrium}

\section{Coupling of the vibrational relaxation model with Navier-Stokes equations}

The implementation of the state-specific inelastic scattering rate in the compressible flow solver is done by adding additional transport equations for every state's population number density, and by transforming the 


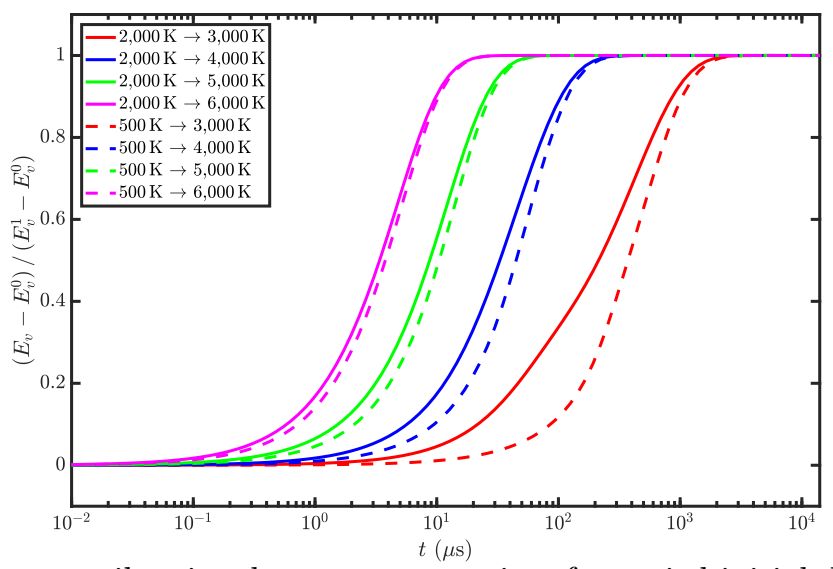

Figure 4: Average vibrational energy versus time for varied initial distributions.

energy conservation equation to account for V-T energy transfer modes.

We call $\dot{\phi}_{k}$ the source term of $\phi_{k}$, number density of vibrational level $k$. The transport equation of the population number density for the m-th vibrational energy state $\phi_{m}$ is defined as :

$$
\frac{\partial \rho \phi_{m}}{\partial t}+\frac{\partial \rho u_{i} \phi_{m}}{\partial x_{i}}=\frac{\partial}{\partial x_{i}} \rho D \frac{\partial \phi_{m}}{\partial x_{i}}+\dot{\phi}_{m} .
$$

$\dot{\phi}_{m}$ is directly computed from the state-specific rates :

$$
\dot{\phi}_{m}=\rho^{2} \sum_{i} \sum_{j} \sum_{k} \sum_{l} \epsilon_{m, i j k l} \times k_{s}\left(v_{i} \rightarrow v_{k}, v_{j} \rightarrow v_{l}, T\right) \times \phi_{i} \times \phi_{j},
$$

where $\epsilon_{m, i j k l}$ characterizes the impact a particular reaction would have on the m-th level population, and is defined as :

$$
\epsilon_{m, i j k l}=-\delta_{i, m}-\delta_{j, m}+\delta_{k, m}+\delta_{l, m} .
$$

While energy gets pumped in/out of the gas molecules vibrational motion during relaxation, energy conservation ensures that an equal amount is transferred from/into the two other modes of internal energy: the translation and rotational modes. We call $E_{t}$ the sum of the translational, rotational and kinetic energies which are assumed at local thermodynamic equilibrium. The coupling with Navier-Stokes equations appears in the right hand side of $E_{t}$ transport equation. All the source terms of states populations number densities $\phi_{k}$ are multiplied by their respective vibrational level energy $e_{v, k}$, and summed up to represent the amount of energy transferred into vibrational motion. The transport equation of $E_{t}$ becomes:

$$
\frac{\partial \rho E_{t}}{\partial t}+\frac{\partial u_{j}\left(\rho E_{t}+P\right)}{\partial x_{j}}=\frac{\partial}{\partial x_{j}} k \frac{\partial T}{\partial x_{j}}-\frac{\partial}{\partial x_{j}}\left(\tau_{i j} u_{i}\right)-\sum_{k} \dot{\phi}_{k} e_{v, m}
$$

where $\rho$ is the fluid density, $u_{i}$ is the velocity vector and $k$ the heat transport coefficient.

In addition to the energy conservation Eq. 26, the compressible solver's governing equations are given by

$$
\begin{gathered}
\frac{\partial \rho}{\partial t}+\frac{\partial \rho u_{j}}{\partial x_{j}}=0, \\
\frac{\partial \rho u_{i}}{\partial t}+\frac{\partial \rho u_{j} u_{i}}{\partial x_{j}}=-\frac{\partial P}{\partial x_{i}}+\frac{\partial \tau_{i j}}{\partial x_{j}}
\end{gathered}
$$

where $P$ is the pressure, and $\tau_{i j}$ is the Newtonian stress tensor, where the kinetic viscosity is computed using Sutherland's law. 


\section{Reduced formulation of the state-specific rates}

In an effort to reduce the computational cost of rates calculations at every iteration, $\dot{\phi}_{m}$ does not need to be directly computed as in Eq. 21. Instead, it is reduced into a more compact form during the initialization of the simulation. In Eq. 24, the summations on the index $k$ and $l$ corresponding to the product of the scattering reaction rate can be pre-computed into a matrix of size number of levels power 3 called $\mathcal{R}_{m i j}$ defined as :

$$
\mathcal{R}_{m i j}=\sum_{k} \sum_{l} \epsilon_{m, i j k l} \times k_{s}\left(v_{i} \rightarrow v_{k}, v_{j} \rightarrow v_{l}, T\right) .
$$

Then, Eq. 24 simply becomes

$$
\dot{\phi}_{m}=\rho^{2} \sum_{i} \sum_{j} \mathcal{R}_{m i j} \times \phi_{i} \times \phi_{j} .
$$

It can be observed that this reduced formulation is analogous to the law of mass action for conventional chemical reaction rates. For each vibrational energy level $m, \mathcal{R}_{m i j}$ gives at a given temperature a clear vision of the kind of trajectory replenishing/depleting the number density $\phi_{m}$. Figure 5 presents $\mathcal{R}_{m i j}$ for the first 9 levels $\left(m=\left[\begin{array}{ll}0 & 8\end{array}\right]\right)$ at a temperature of $3000 \mathrm{~K}$. Dark blue corresponds to a maximum depletion, and dark red to a maximum replenishment over the whole rates temperature range (2000 to $4000 \mathrm{~K}$ ). The table is symmetric along the identity diagonal. A first observation is that all almost all cells located on the $i=m$ or $j=m$ lines are blue, i.e. collisions involving at least one molecule of level $m$ usually result in a depletion of $\phi_{m}$. At high levels $(m>3)$, depletion is usually maximum when both colliding molecules are initially on level $m$. On the other hand, it tends to be shifted towards the right/left of the $(i, j)=(m, m)$ cell for lower

levels as inelastic collisions with a $m$ molecule are more likely to occur when involving a partner at higher level. This appears clearly when looking at the $m=2$ table for instance. At $3000 \mathrm{~K}$, a $(i, j)=(2,2)$ collision is less effective at depleting $\phi_{2}$ than $(i, j)=(6,2)$ for instance. Interestingly, the ground state rates table is characterized by a positive production (red cell) for any neighbor of the $(i, j)=(0,0)$ cell. Similarly, the highest replenishment rates are found for high levels $(m>3)$ in vicinity of the $(m, m)$ cell on its diagonals. Logically, the fourth quadrant (both $i, j>m$ ) always have higher rates than the second quadrant (both $i, j<m)$.

\section{Turbulent planar jet simulations}

\section{A. Numerical configurations}

The simulations were performed using the in-house compressible flow solver, UTComp, which has been extensively verified and validated..$^{78-20}$ The solver uses a finite difference fifth-order WENO LLF scheme with characteristics reconstruction to compute the fluxes, ${ }^{21,22}$ while a fourth-order central scheme is used for the viscous and diffusion terms. The viscosity is determined using Sutherland's law with $\mathrm{N}_{2}$ coefficients and the thermal diffusivity is obtained using a constant Prandtl number of 0.72. Further details on the flow solver are provided in Koo. ${ }^{21}$ Time-integration is carried out using a fourth-order Runge-Kutta scheme. The planar jet has a height of $8 \mathrm{~mm}$ and is $1.6 \mathrm{~cm}$ wide, while the domain is $16 \mathrm{~cm}$ long. It is discretized using an orthogonal grid system of $\left(n_{x}, n_{y}, n_{z}\right)=(2048,640,128)$ cells. The domain is periodic in the spanwise direction, and non-reflective characteristic boundary conditions are applied at the other boundaries of the domain. Statistics were sampled over $0.5 \mathrm{~ms}$, which corresponds to 1.0 flow-through timescale $\tau_{c}$ based on the integrated centerline velocity. The plane jet inflow is a fully resolved $2 \mathrm{D}$ channel flow and was obtained from an auxiliary DNS. It possesses a $0.8 \mathrm{~mm}$ top and bottom boundary layer, and a $400 \mathrm{~m} / \mathrm{s}$ core velocity, with a static pressure of 2.0 atmosphere and a static temperature of $2000 \mathrm{~K}$ for a Reynolds number based on the boundary layer height $R e_{\lambda}=1875$. The coflow is also at 2.0 atmospheric pressure, has a speed of $80 \mathrm{~m} / \mathrm{s}$ and its static temperature is $4000 \mathrm{~K}$. The simulation ran with a Courant-Friedrichs-Lewy number of roughly 0.9 , giving a timestep of about $0.1 \mu \mathrm{s}$. The code uses domain-decomposition based parallelization, and each simulation was run on 8000 cores for 10 hours. 

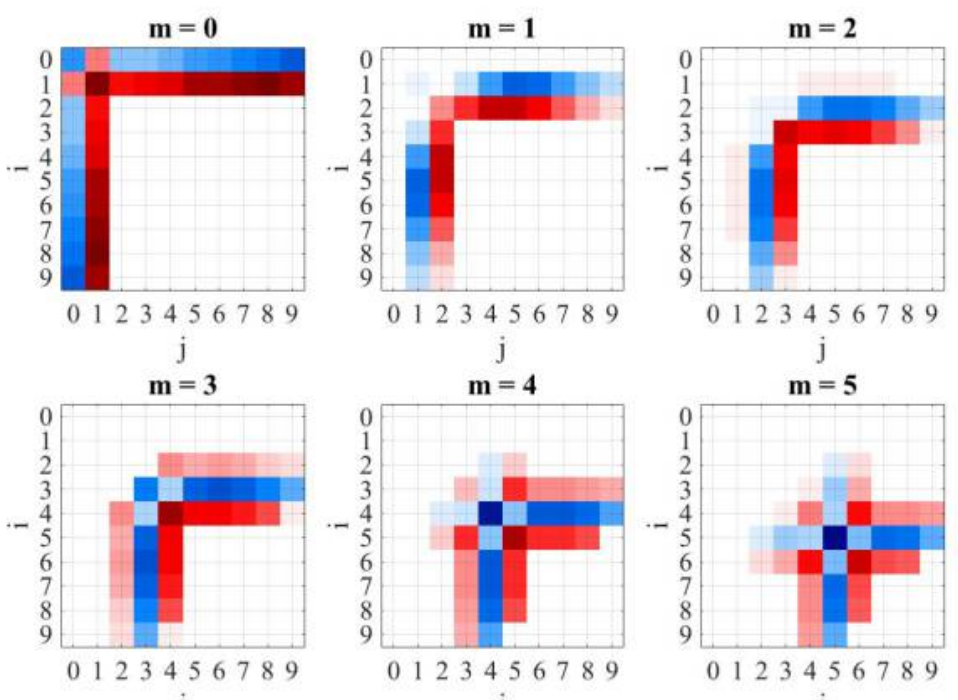

01223456789

0123456789

$\mathbf{m}=6$
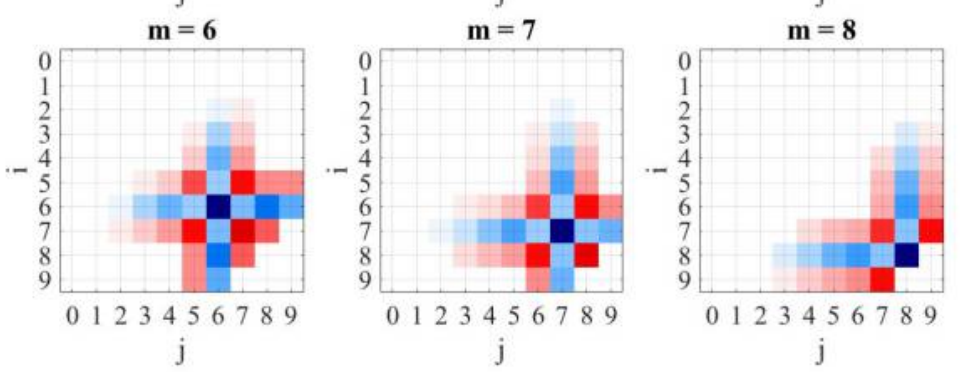

Figure 5: $\mathcal{R}_{m i j}$ for the first 9 levels $\left(m=\left[\begin{array}{l}0 \\ \text { 8 }\end{array}\right]\right.$ ) at a temperature of $3000 \mathrm{~K}$.

\section{B. Turbulent planar jet}

A numerical simulation of a $\mathrm{N}_{2}$ turbulent plane jet configuration described in section III was carried out. An inert mixture fraction $Z_{m i x}$ was transported alongside the reacting first 10 vibrational state number densities. $Z_{m i x}$ allows us to observe how turbulent mixing tends to naturally bring the cell-averaged vibrational energy state distribution out of the equilibrium. The vibrational state distribution is transported and resolved using the reaction rates previous determined in Sec.II to account for nonequilibrium and the distribution relaxation process. Figure 6 presents an instantaneous snapshot of the density gradient magnitude and translation temperature fields, revealing a highly turbulent mixing layer and post-potential core region.
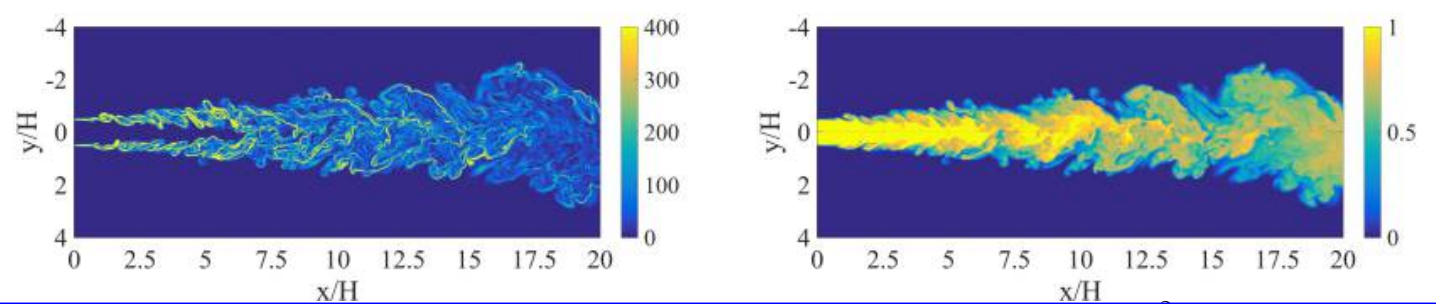

Figure 6: Instantaneous snapshots of (top) Magnitude of density gradient in $\mathrm{kg} / \mathrm{m}^{2}$ of the $\mathrm{N}_{2}-\mathrm{N}_{2}$ planar jet and (bottom) contour of inert scalar $Z_{m i x}$.

Instantaneous snapshots of $\phi_{1}$ and $\phi_{9}$ are shown in Fig. 7. It can be seen that the overall mixing appears to be complex and dependent on the vibrational level. $\phi_{9}$ appears to be dominated by the cold part of the jet while lower level populations like $\phi_{1}$ seem to mix more evenly, similarly to $Z_{m} i x$. This suggests that is $\phi_{1}$ relaxation process is slower than the mixing timescale, meaning the mixing process dominates. On the contrary, at higher level 9 known to have the highest scattering rates (i.e. relaxation faster than mixing), 
the state population doesn't simply follow the same process.
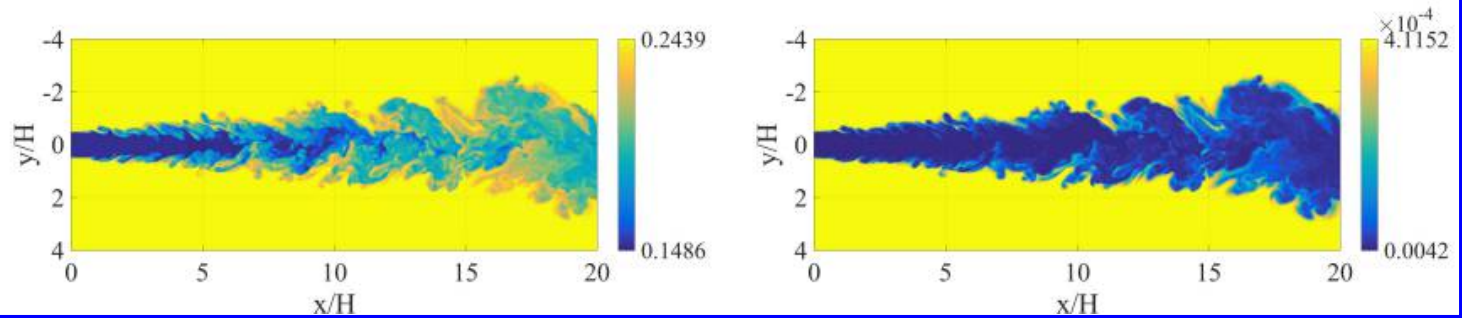

Figure 7: Instantaneous snapshots of vibrational state population number densities for levels $\imath=1$ (left) and $i=9$ (right).

Before further analysis on the state populations, it is important to quantify the amount of vibrational nonequilibrium triggered by the turbulent mixing between the cold and hot flows. Figure 8 shows the error between the integrated bulk vibrational energy of the flow $E_{v}$ compared to its local equilibrium value $E_{v}{ }^{*}$ computed assuming a Boltzmann distribution at the local temperature $T$. It appears the the mixing layer is overwhelmingly vibrationally under-excited, with peak departure from equilibrium of only $3 \%$. This value is integrated over the whole energy distribution and therefore dominated by the most-populated levels 0 and 1.

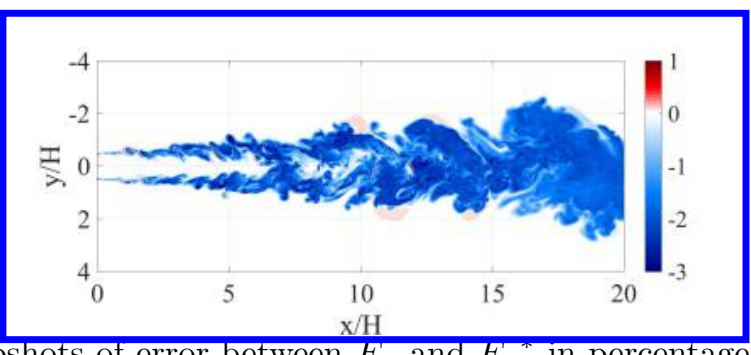

Figure 8: Instantaneous snapshots of error between $E_{v}$ and $E_{v}$ in percentage. Red/blue indicate a locally vibrationally over/under-excited population.

The state-specific departure from equilibrium for level $i$ is quantified by a parameter called $\mathcal{B}_{i}\left(\mathrm{E}_{\mathrm{v}}\right)$ defined as :

$$
\mathcal{E}_{i}=\frac{\phi_{i}-\mathcal{B}_{i}\left(\mathrm{E}_{\mathrm{v}}\right)}{\mathcal{B}_{i}\left(\mathrm{E}_{\mathrm{v}}\right)}
$$

where $\mathcal{B}_{i}\left(\mathrm{E}_{\mathrm{v}}\right)$ is the number density of level $i$ for a Boltzmann distribution yielding the same bulk $E_{v}$. Figure 9 shows the magnitude of that factor $\mathcal{E}_{i}$ throughout the computational domain on the right, along with its distribution with local mixture fraction $Z_{m i x}$ on the left. Only levels 1,2, 3 and 9 are shown for conciseness reasons, as levels 3 to 9 behave similarly.

First, the higher the vibrational level $i$, the higher $\mathcal{E}_{i}$ becomes. This indicates that the level is locally over-populated. Level 1 is on the other hand slightly locally under-populated, which is consistent with the observation previously made from Fig. 8 showing a bulk under-excited vibrational energy $E_{v}$. According to the rates definition and thermal bath tests, the highest levels adjust faster than the lowest one to the local thermal equilibrium conditions. As the local relaxation timescale of the high levels becomes smaller than the mixing timescale, these levels relax towards the local equilibrium $E_{v}{ }^{*}$ (larger than $E_{v}$ ) by increasing their population. This results in a relative under-population of the slowly relaxing level 1 compared to $\mathcal{B}_{1}\left(\mathrm{E}_{\mathrm{v}}\right)$. $\mathcal{E}_{9}$ high values are attributed to the large range of $\phi_{9}$ throughout the mixing layer. Possibly, one intermediary level could have a relaxation timescale comparable to the mixing timescale. As observed in the experiment of Reising et al. ${ }^{23}$ there is an inflection point in the shear layer where the flow switches from under to over-populated vibrational modes. This happens when the mixing and relaxation timescales become similar and no process dominates the other. In the present study, a similar phenomenon affects the distribution of $\phi_{2}$. It is therefore consistent to observe temperature inversion on one state only, and to notice that this state separates those exclusively over-excited and under-excited.

Similar observations can be made when looking at time-averaged y-profiles at a downstream location of $x=2 H$. Figure 10 shows the various $\mathcal{B}_{i}\left(\mathrm{E}_{\mathrm{v}}\right)$ for $i \in\left[\begin{array}{lll}1 & 2 & 3\end{array}\right]$ from left to right, with the temperature profile 

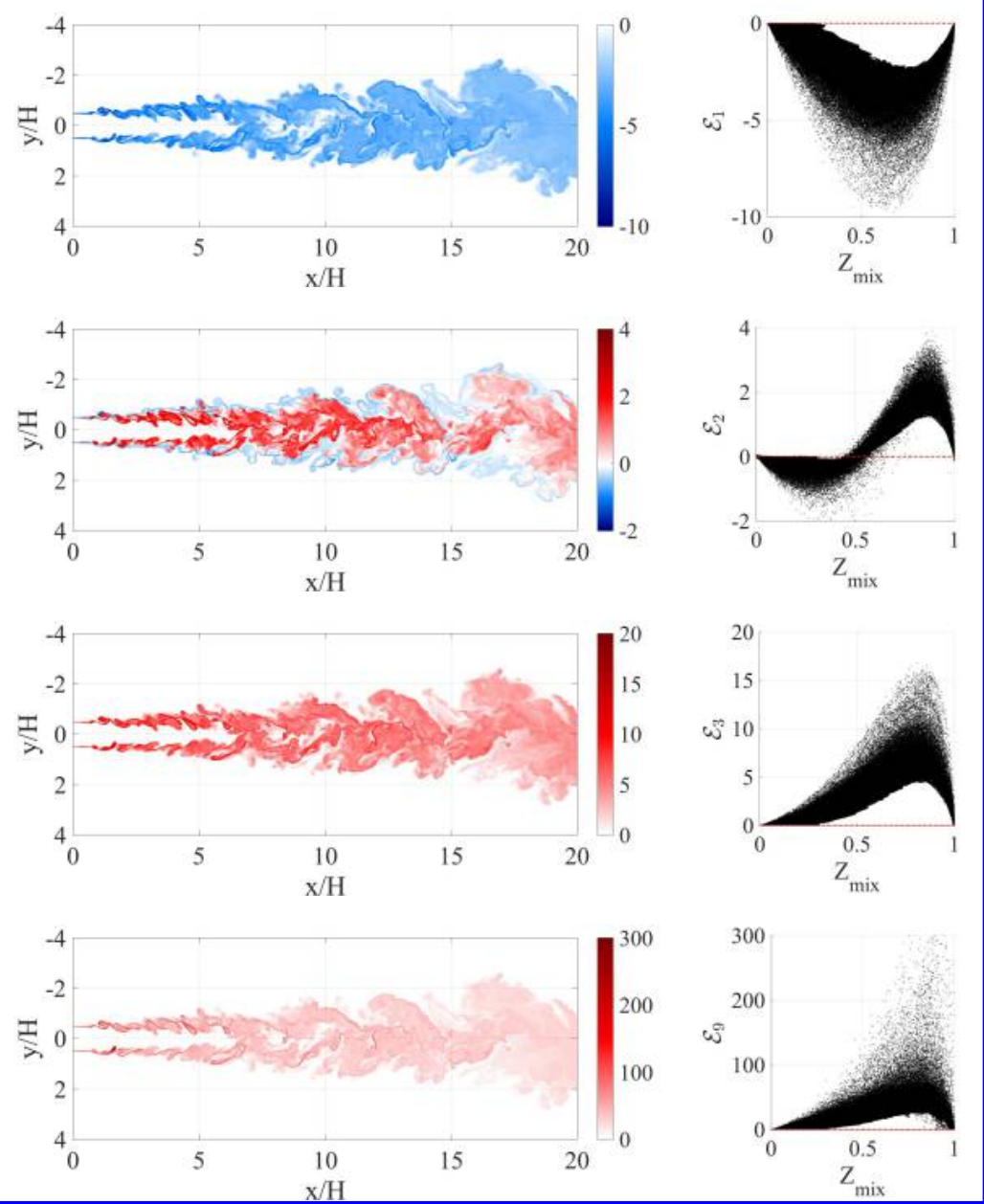

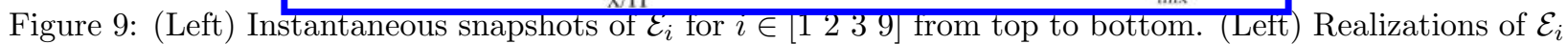
for $i \in\left[\begin{array}{llll}1 & 2 & 3 & 9\end{array}\right]$ from top to bottom with $Z_{m i x}$.
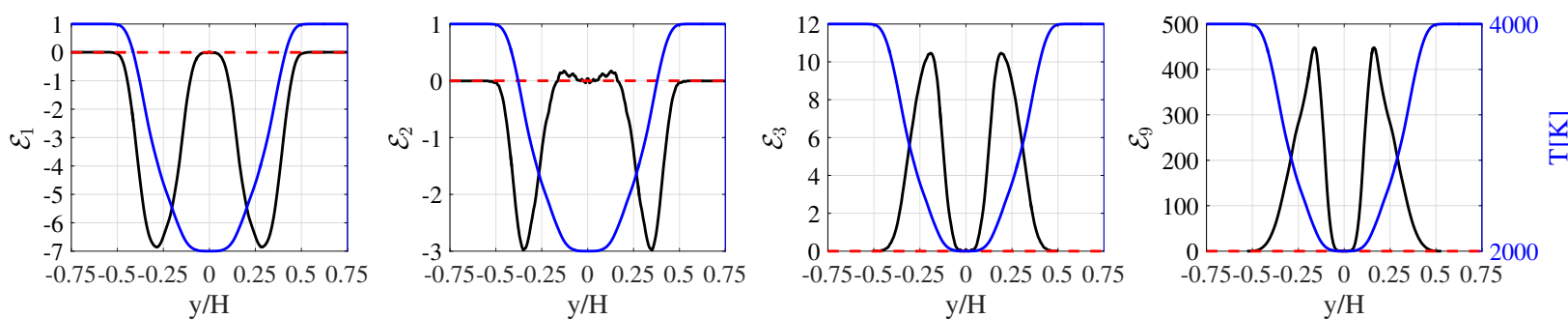

Figure 10: Time-averaged Y-profiles at $\mathrm{x}=2 \mathrm{H}$ of $\mathcal{E}_{i}$ for $i \in\left[\begin{array}{lll}1 & 2 & 3\end{array}\right]$ from left to right.

in blue on the second y-axis. It can be noticed that the location of the peak error changes as the level increases. While closely matching the center of the shear layer $(\mathrm{T}=3000 \mathrm{~K})$ for the level 1 , it is strongly skewed towards the centerline for level 9 which is again attributed to the larger ratio of states population densities $\phi_{i}$ with increasing level $i$. The direct impact of the relaxation rates on these profiles is therefore difficult to extract, as it is blended within the mixing-triggered nonequilibrium. It is therefore interesting to compare the populations with what their local value would be using the mixture fraction to compute a $\phi_{\operatorname{mix}}$ distribution simply defined as : $Z_{m i x} \times \phi(T=2000 \mathrm{~K})+\left(1-Z_{m i x}\right) \times \phi(T=4000 \mathrm{~K})$, corresponding to a frozen V-V and V-T energy exchange process. Similarly, $T_{m i x}$ is defined as the linear mix of jet and coflow temperatures. First, Fig. 11 verifies a near-perfect collapse between $T$ and $T_{m i x}$ y-profiles. Then, Fig. 12 presents for the same 4 levels as before both $\phi_{i}$ (left y-axis) and $\mathcal{E}_{i}$ (right axis). The simulation profiles are shown in solid lines while the profiles obtained using the inert mixture fraction are dashed. Logically, the 
rates tend to preserve a Boltzmann-type vibrational energy distribution and limit the errors $\mathcal{E}_{i}$ compared to the inert mixing case (for instance, the error $\mathcal{E}_{9}$ is about five times lower).

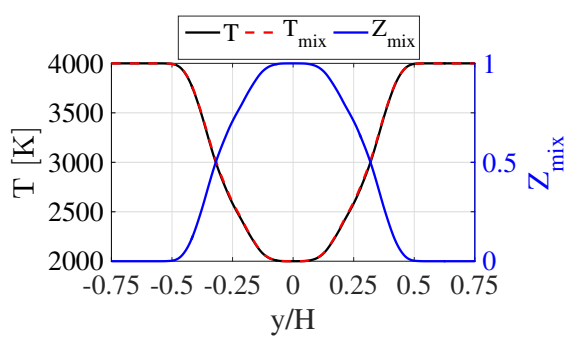

Figure 11: Time-averaged Y-profiles at $\mathrm{x}=2 \mathrm{H}$ of temperature $\mathrm{T}$, temperature $T_{m i x}$ obtained from the mixture fraction, and mixture fraction $Z_{m i x}$.
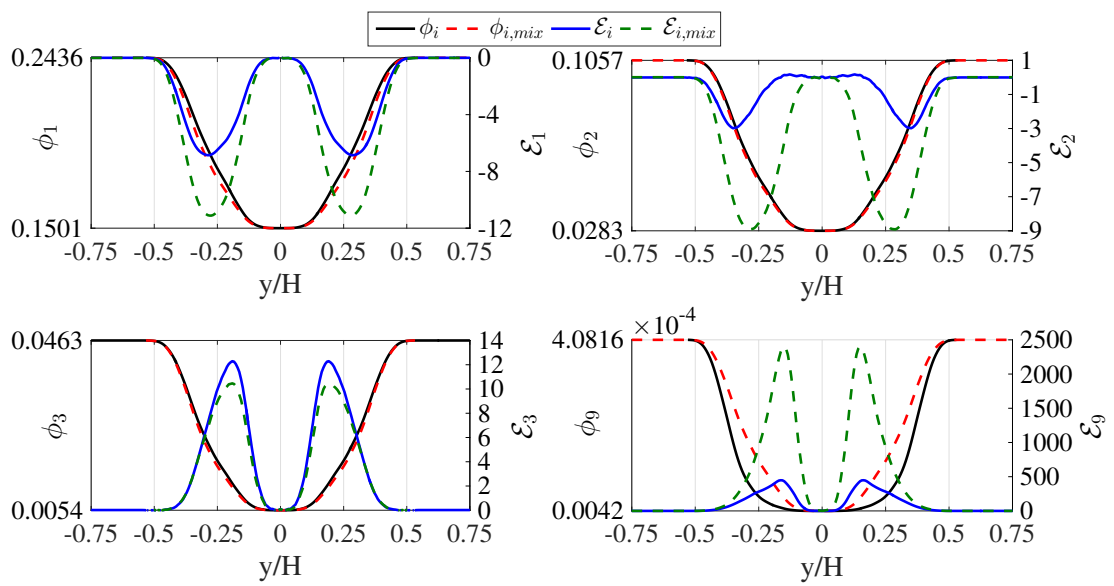

Figure 12: Time-averaged Y-profiles at $\mathrm{x}=2 \mathrm{H}$ of $\phi_{i}$ and $\phi_{i, m i x}$ obtained from the mixture fraction (left axis), alongside $\mathcal{E}_{i}$ and $\mathcal{E}_{i, \text { mix }}$ obtained from the mixture fraction (right axis).

\section{Conclusions}

The QCT method was used to calculate a set of vibrational state-specific scattering rates for the collision $\mathrm{N}_{2}\left(v_{1}\right)+\mathrm{N}_{2}\left(v_{2}\right)$. For this system, the maximum temperature was approximately $4,000 \mathrm{~K}$, so only the first 10 vibrational states were considered (the likelihood of higher vibrational states is very small). During the QCT simulation, the translational-rotational energy was sampled from one of nine temperatures ranging from $500 \mathrm{~K}$ to $6,000 \mathrm{~K}$. In total, 2.8 billion trajectories were simulated to calculate the set of rates.

A numerical simulation of turbulent plane jet coupled with a vibrational state populations solver was then performed to investigate the coupling between turbulence mixing and vibrational nonequilibrium. It was found that the mixing between the cold jet and hot coflow would generate a vibrationally under-excited mixing layer. However the highest vibrational states are on the contrary drastically over-excited, which would potentially affect chemical reactions. A numerical description of nonequilibrium only resolving the bulk vibrational energy, i.e. integrated over the whole energy level distribution, would not be sufficient for reacting flows.

\section{Acknowledgement}

This work is supported financially through AFOSR BRI Award No. FA9550-12-1-0460 with Dr. Ivett Leyva as Program Manager. Philip Varghese gratefully acknowledges support of a Moncrief Fellowship that enabled extended visits to the University of Michigan in Spring 2016. The authors also gratefully acknowledge the generous allocation of computing time by the Texas Advanced Computing Center and NASA High-End Computing (HEC) Program through the NASA Advanced Supercomputing (NAS) Division at Ames Research Center. 


\section{References}

${ }^{1}$ Knab, O., Gogel, T. H., Fruhauf, H. H., and Messerschmid, E. W., "CVCV-model validation by means of radiative heating calculations," 33rd AIAA Aerospace Science Meeting and Exhibit, No. AIAA-95-0623, 1995.

${ }^{2}$ Knab, O., Fruhauf, H. H., and Messerschmid, E. W., "Theory and validation of the physically consistent coupled vibrationchemistry-vibration model," Journal of Thermophysics and Heat Transfer, Vol. 9, No. 2, 1995, pp. 219-226.

>3Park, C., "Assessment of a two-temperature kinetic model for dissociating and weakly ionizing nitrogen," Journal of Thermophysics, Vol. 2, No. 1, 1988, pp. 8-16.

${ }^{4}$ Arsentiev, I. V., Loukhovitski, B. I., and Starik, A. M., "Application of state-to-state approach in estimation of thermally nonequilibrium reaction rate constants in mode approximation," Chemical Physics, Vol. 398, 2012, pp. 73-80.

${ }^{5}$ Voelkel, S., Raman, V., and Varghese, P. L., "Effect of thermal nonequilibrium on reactions in hydrogen combustion," Shock Waves, 2016, pp. 1-11.

${ }^{6}$ Fiévet, R., Voelkel, S., Koo, H., Varghese, P., and Raman, V., "Effect of Thermal Nonequilibrium on Ignition in Scramjet Combustors," Accepted for the 36rd Symposium (International) on Combustion.

${ }^{7}$ Fiévet, R., Koo, H., and Raman, V., "Numerical simulation of a scramjet isolator with thermodynamic nonequilibrium," 22nd AIAA Computational Fluid Dynamics Conference, 22-26th June 2015, Dallas, TX, USA, No. AIAA-2015-3418, 2015.

${ }^{8}$ Reising, H., Kc, U., Voelkel, S., Clemens, N. T., Raman, V., Varghese, P. L., and H., "Vibrational Non-equilibrium Effects in Supersonic Jet Mixing," 52nd AIAA Aerospace Sciences Meeting, 13-17 January 2014, National Harbor, Maryland, No. AIAA 2014-0231, 2014.

${ }^{9}$ Koo, H., Raman, V., and Varghese, P., "Direct numerical simulation of supersonic combustion with thermal nonequilibrium," Proceedings of the Combustion Institute, Vol. 35, No. 2, 2015, pp. 2145-2153.

${ }^{10}$ Taylor, R., Camac, M., and Feinberg, R., "Measurements of vibration-vibration coupling in gas mixtures," Symposium (International) on Combustion, Vol. 11, No. 1, 1967, pp. 49 - 65.

${ }^{11}$ Landau, L. D. and Teller, E., "Theory of Sound Dispersion," Phys. Z. Sowjetunion, Vol. 10, 1936.

${ }^{12}$ Bender, J. D., Valentini, P., Nompelis, I., Paukku, Y., Varga, Z., Truhlar, D. G., Schwartzentruber, T., and Candler, G. V., "An improved potential energy surface and multi-temperature quasiclassical trajectory calculations of $\mathrm{N}_{2}+\mathrm{N}_{2}$ dissociation reactions," The Journal of Chemical Physics, Vol. 143, No. 5, 2015, pp. 054304.

$\checkmark{ }^{13}$ Voelkel, S., Varghese, P. L., and Raman, V., "Quasi-State-Specific QCT Method for Calculating the Dissociation Rate of Nitrogen in Thermal Non-Equilibrium," 54th AIAA Aerospace Sciences Meeting, 4-8 January 2016, San Diego, California, USA, No. AIAA 2016-0449, 2016.

${ }^{14}$ Prince, P. and Dormand, J., "High order embedded Runge-Kutta formulae," Journal of Computational and Applied Mathematics, Vol. 7, No. 1, Mar 1981, pp. 67-75.

${ }^{15}$ Paukku, Y., Yang, K. R., Varga, Z., and Truhlar, D. G., "Global ab initio ground-state potential energy surface of $\mathrm{N}_{4}$," The Journal of Chemical Physics, Vol. 139, No. 4, 2013, pp. 044309.

${ }^{16}$ Henriksen, N. E. and Hansen, F. Y., Theories of Molecular Reaction Dynamics: The Microscopic Foundation of Chemical Kinetics, Oxford Graduate Texts, Oxford University Press, 2012.

17 Millikan, R. C. and White, D. R., "Systematics of vibrational relaxation," Journal of Chemical Physics, Vol. 39, 1963, pp. 3209.

${ }^{18}$ Koo, H., Donde, P., and Raman, V., "A Quadrature-based LES/Transported Probability Density Function Approach for Modeling Supersonic Combustion," Proceedings of the Combustion Institute, Vol. 33, 2011, pp. 2203-2210.

$\checkmark{ }^{19}$ Koo, H., Donde, P., and Raman, V., "LES-based Eulerian PDF approach for the simulation of scramjet combustors," Proceedings of the Combustion Institute, Vol. 34, No. 2, 2013, pp. 2093-2100.

$\checkmark{ }^{20}$ Donde, P., Koo, H., and Raman, V., "A multivariate quadrature based moment method for LES based modeling of supersonic combustion," Journal of Computational Physics, Vol. 231, No. 17, 2012, pp. 5805-5821.

${ }^{21}$ Koo, H., Large-eddy simulations of scramjet engines, Ph.D. thesis, The University of Texas at Austin, 2011.

${ }^{22}$ Jiang, G.-S. and Shu, C.-W., "Efficient Implementation of Weighted ENO Schemes," Journal of Computational Physics, Vol. 126, 1996, pp. 202-228.

${ }^{23}$ Reising, H., Haller, T., Clemens, N., Varghese, P., Fiévet, R., and Raman, V., "Spontaneous Raman Scattering Temperature Measurements and Large Eddy Simulations of Vibrational Non-equilibrium in High-Speed Jet Flames," 32nd AIAA Aerodynamic Measurement Technology and Ground Testing Conference, 13-17 June 2016, Washington, D.C., No. AIAA 20163144,2016 . 
This article has been cited by:

1. Daniil Andrienko, Iain D. Boyd. Master equation simulation of O2-N2 collisions on an ab-initio potential energy surface . [Citation] [PDF] [PDF Plus] 